\title{
Treating Mechanical Dysfunctions of Lower Back through Lumbar Spine Assessment of Structural Diagnosis and Management (SDM): A New Comprehensive Approach of Musculoskeletal Medicine
}

\author{
KM Amran Hossain ${ }^{1}$, Mohammad Shahadat Hossain ${ }^{2}$, Sapia Akter ${ }^{3}$, Mohammad Anwar Hossain ${ }^{4}$ \\ and Md Obaidul Haque ${ }^{1 *}$ \\ ${ }^{1}$ Department of Physiotherapy, Bangladesh Health Professions Institute, Bangladesh \\ ${ }^{2} J a h a n g i r n a g a r$ University, Bangladesh \\ ${ }^{3}$ Agrani Specialized Physiotherapy Centre, Bangladesh \\ ${ }^{4}$ Centre for the Rehabilitation of the Paralyzed, Bangladesh
}

*Corresponding author: Obaidul Haque, Head of Physiotherapy Department and Vice-principal, Bangladesh Health Professions Institute (BHPI), Bangladesh

To Cite This Article: Md Obaidul Haque, Treating Mechanical Dysfunctions of Lower Back through Lumbar Spine Assessment of Structural Diagnosis and Management (SDM): A New Comprehensive Approach of Musculoskeletal Medicine. Am J Biomed Sci \& Res. 2020 - 7(1). AJBSR. MS.ID.001101. DOI: 10.34297/AJBSR.2020.07.001101.

Received: 眥 December 02, 2019 ; Published: 眥 January 17, 2020

\begin{abstract}
Low back pain and radiculopathies are leading health issues and mainly managed through conservative approaches concentrating on articular and neural structures of lumbar spinal segments. Although more than one-third of the sources causing lumbar pain, radiculopathy or associated impairments may not arise from the lumbar spine, articulations, nerve roots or local structures. Structural Diagnosis and Management is a comprehensive and systematic approach of taking history, examination of arthokinemetic and osteokinemtic motions of lumbar spine, stretching the series of muscles in lumbopelvic and lower extremities with biomechanical rationale, strength test to relative prime, segmental and regional stabilizer, neurological examination to the biomechanical contributor, myotomes and isolated neural structures to generate a diagnosis. The concept is developed by Dr. M Shahadat Hossain with a hypothesis that, disc herniation, degenerative disc disease or other structural and mechanical phenomenon might be treated creating a biomechanical correction by strengthening the week structures and stretching the adherent or spasmodic structures with or without localized release to the adherent neural sensitivity. The concept integrates articular, contractile, inert structural dysfunctions concentrating on spinal, regional and biomechanical connections to ensure assessment and treatment through the appropriate clinical reasoning process.
\end{abstract}

Keywords: SDM; Lumbar spine assessment; Low back pain

\section{Introduction}

Low back pain is a prominent musculoskeletal condition that affects health and impacts upon the social and economic status of any population worldwide [1]. Hoy, Brooks, Blyth, and Buchbinder [2] avowed more than three fourth of global populations suffer low back pain anytime in their life. Low back pain is manifested by several structural involvements of the lumbosacral spine as, the inter vertebral disc, facet joint, sacroiliac joint, spinal body and arches, neural tissues and surrounding soft tissues [3]. Lumbar spine assessment needs a comprehensive evaluation of contractile and non-contractile structures that contribute to the mechanical pain, structural dysfunction or even neurological symptoms that might manifest differently over time [4]. In lumbar spine assessment, the conventional protocol and approaches focus on the lumbar region, 
spinal neural structures or the nerve roots that are being compressed or even adherent or hypo-mobile structures in the lumbar area [5]. There are shreds of evidence that suggest nearly one-third of the sources causing lumbar pain, radiculopathy or associated impairment may not arise from the lumbar spine, articulations, nerve roots or local structures. There might be a contribution of local or regional muscles, inert structures that leads chronic biomechanical abnormalities anywhere in the human body causing symptoms in lower back [6]. There is a hypothesis that disc herniation, degenerative disc disease or other structural and mechanical phenomena might be treated creating a biomechanical correction by strengthening the week structures and stretching the adherent or spasmodic structures with or without localized release to the adherent neural sensitivity [7].

\section{Structural Diagnosis and Management (SDM)}

Structural Diagnosis and Management (SDM) of musculoskeletal medicine focuses on a comprehensive approach to assess both the contractile and non-contractile structures to generate a hypothesis to solve the specific sources hindering normal activities in the lumbar spine. This has been developed through 15 years' experience of Dr. M Shahadat Hossain treating diverse cases of musculoskeletal medicine. The SDM assessment directs conservative management procedure in a way of taking history, examination of arthokinemetic and osteokinemtic motions of lumbar spine, stretching the series of muscles in lumbopelvic and lower extremities with biomechanical rationale, strength test to relative prime, segmental and regional stabilizer, neurological examination to the biomechanical contributor, myotomes and isolated neural structures to generate a diagnosis (Annexure 1).

The assessment procedure begins with some subjective information and cues, history and duration. The body chart represents a graphical presentation of symptom and posture in anterior, lateral and posterior aspects. The kinetic and kinematic motions are also assessed accordingly with the screening of other relative key points in the integrated body system. The passive stretch test evaluates the pain and tightness for extensibility and strength test examines the strength of muscles in lumbopelvic and lower extremities with biomechanical rationale. Neurological examination examines the myotomes, dural signs, and specific neural sensitive structures. The clinical examination comprises the radiological examination to conclude to a diagnosis.

The diagnosis is categorized as muscular dysfunction, muscular and neurological dysfunction or complete mechanical dysfunction of the lumbar spine. The treatment approach in SDM focuses primarily on stretching or releasing the adherent or neural sensitive structures to create a facilitating environment to enhance the bodily healing process, strengthening or restoring the functions of contractile structures, regional and biomechanical stabilizers and maintenance of normal static or dynamic biomechanical alignment.

\section{Discussion}

The assessment breaks the barrier to think only the lumbar spine or only disc to be the only structures to be treated for mechanical dysfunctions of lower back. The lower body quadrant, trunk or even cervical-thoracic orientation can contribute to Low back symptoms or radicular pain in the lower limb [8], the assessment opens a window of thought to include these in a comprehensive assessment and treatment. Moreover, the conventional approaches have limitation in manual therapy techniques apart of lumbar hypermobility, dural involvement or an affirmative Straight leg raise, the SDM approach can reach to each part of neural sensitive structures that are proven to be responsible to create chronic central or structural sensitivity [9], causing recurrence or chronicity of LBP. The assessment and treatment techniques have been disseminated by more than 15 short courses to 246 practicing physiotherapists in Bangladesh and a couple of research projects are on progress. The mastermind behind the concept Dr. M Shahadat Hossain aims to disseminate globally with a strong rationale of a comprehensive approach to deal Lumbar spine issues with a shorter duration of management, clinical reasoning, and fewer recurrence episodes.

\section{Conclusion}

Structural Diagnosis and Management (SDM) of the lumbar spine is a comprehensive approach to diagnosis and treatment of low back pain and associated radiculopathies in conservative approach for physiotherapy and healthcare professionals. The concept integrates articular, contractile, inert structural dysfunctions concentrating on spinal, regional and biomechanical connections to ensure proper assessment and treatment through the appropriate clinical reasoning process.

\section{Acknowledgement}

Authors acknowledge Dr. Nasirul Islam and Md. Arifur Rahman for their continues supports in developing the assessment

\section{Funding and Copyright}

The SDM assessment is a copyright protected to the Institute of Advanced Mechanical Correction Therapy.

\section{References}

1. Woolf AD, Pfleger B (2003) Burden of major musculoskeletal conditions. Bull World Health Organ 81(9): 646-656.

2. Hoy D, Brooks P, Blyth F, Buchbinder R (2010) The epidemiology of low back pain. Best Pract Res Clin Rheumatol 24(6): 769-781.

3. Petersen T, Laslett M, Juhl C (2017) Clinical classification in low back pain: best-evidence diagnostic rules based on systematic reviews. BMC Musculoskelet Disord 18(1): 188.

4. Booth J, Moseley GL, Schiltenwolf M, Cashin A, Davies M, et al. (2017) Exercise for chronic musculoskeletal pain: a biopsychosocial approach. Musculoskeletal care 15(4): 413-421.

5. Meyer S, Harrison D (2018) The Mc Kenzie Method and Treatment of Low Back Pain. 
6. Allegri M, Montella S, Salici F, Valente A, Marchesini M, et al. (2016) Mechanisms of low back pain: a guide for diagnosis and therapy. F1000 Res 5.

7. Hall T, Robinson K (2015) Neuro dynamics: when and why? Oxford Textbook of Musculoskeletal Medicine 26: 143.

8. Neto T, Freitas SR, Marques M, Gomes L, Andrade R, et al. (2017) Effects of lower body quadrant neural mobilization in healthy and low back pain populations: a systematic review and meta-analysis. Musculoskelet Sci Pract 27: 14-22.

9. Mitra M, Gaikwad N (2019) The Effect of Straight Leg Raise with Lumbar Stabilization Exercises in Subjects of Low Back Pain with Adverse Neural Tension between the Age Group 20-50 Years. Indian Journal of Physiotherapy \& Occupational Therapy 1: 13(1). 